\title{
煤基球形多孔碳用于锂离子电池负极材料的性能研究
}

\author{
李君，曹亚丽，王鲁香，贾殿赠
}

(新疆大学 能源材料化学教育部重点实验室, 先进功能材料新疆维吾尔自治区重点实验室, 应用化学研究所, 乌鲁 木齐 830046)

摘 要: 因具有较短的锂离子扩散路径、大的比表面积等优势, 球形碳材料在锂离子电池负极材料中展露出良好的 应用前景。研究以新疆库车产煤为原料, 采用电弧放电法及化学活化法制备出了具有多孔结构的煤基球形碳。通过 $\mathrm{X}$ 射线衍射(XRD)、扫描电镜(SEM)、拉曼光谱(Raman)、氮气吸脱附法和恒电流充放电等测试手段对材料结构、 形貌和电化学性能进行了表征。结果表明, 在 $100 \mathrm{~mA} / \mathrm{g}$ 的电流密度下, 煤基球形多孔碳的首次放电比容量可达到 $1188.9 \mathrm{mAh} / \mathrm{g}$, 远高于商业石墨负极 $372 \mathrm{mAh} / \mathrm{g}$ 的理论比容量。此外, 该材料还表现出了良好的循环稳定性, 经历 200 圈循环后的放电比容量为 $844.9 \mathrm{mAh} / \mathrm{g}$ 。煤基球形多孔碳优异的电化学性能得益于活化过程所产生的分级孔道 结构能为锂离子提供更多储存空间，从而提高了电极的容量及循环稳定性。

关 键 词: 煤基; 球形多孔碳; 锂离子电池; 负极

中图分类号: O643 文献标识码: A

\section{Performance of Coal-derived Spherical Porous Carbon as Anode Materials for Lithium Ion Batteries}

\author{
LI Jun, CAO Ya-Li, WANG Lu-Xiang, JIA Dian-Zeng
}

(Key Laboratory of Energy Materials Chemistry, Ministry of Education, Key Laboratory of Advanced Functional Materials, Autonomous Region, Institute of Applied Chemistry, Xinjiang University, Urumqi 830046, China)

\begin{abstract}
Spherical porous carbon nanomaterials is a promising candidate as anode materials for next-generation LIBs due to its large specific surface area which decreases the lithium ion transport distance. Spherical porous carbon nanomaterials with uniform morphology were prepared by arc-discharging followed by chemical activation using coal as precursor. The as-prepared spherical porous carbon samples were characterized by X-ray diffraction (XRD), scanning electron microscope (SEM), Raman spectrope (Raman), the nitrogen adsorption-desorption, galvanostatic charge and discharge method and so on. The result indicated that the spherical porous carbon materials have high specific capacity of $1188.9 \mathrm{mAh} / \mathrm{g}$, which exceeds the commercial mesophase carbon spheres (372 mAh/g). Moreover, the spherical porous carbon nanomaterials also afford excellent cyclic stability and a reversible capacity of $844.9 \mathrm{mAh} / \mathrm{g}$ remains ever after 200 charge/discharge cycles at current density of $100 \mathrm{~mA} / \mathrm{g}$. The excellent electrochemical performance of coal based spherical porous carbon may be originated from the graded pore structure, which can provide more storage space for lithium ion, thus improving the capacity and cycle stability of the electrode.
\end{abstract}

Key words: coal-derived; sphere porous carbon; lithium ion battery; anode

收稿日期: 2016-11-25; 收到修改稿日期：2017-02-22

基金项目：国家自然科学基金(U1203292); 中国科学院西部之光人才培养计划(YBXM-2014-07)

National Science Foundation of China (U1203292); Western Light Program of Talent Cultivation of the Chinese Academy of Sciences (YBXM-2014-07)

作者简介: 李 君(1982-), 女, 博士研究生. E-mail: Junli107@163.com

通讯作者: 贾殿赠, 教授.E-mail: jdz@xju.edu.cn 
锂离子电池是当今社会最为重要的二次电池, 具有比容量高、工作电压高、循环寿命长、无记忆 效应和对环境友好等优点, 是高容量、大功率电池 的理想之选 ${ }^{[1-2]}$ 。目前, 商业化锂离子电池使用的负 极材料主要包括石墨、可石墨化碳和无定形碳等碳 材料。其中, 无定形碳材料因具有大的比表面积及 开放的纳米级孔洞和通道, 使其容量得以大幅度提 高, 而且具有可以忽略的体积膨胀率, 引起研究者 们的极大兴趣 ${ }^{[3]}$ 。但在实际应用中, 无定形碳材料较 低的振实密度严重影响了电极的加工过程, 涂布后 容易掉粉, 影响电化学性能, 制备球形碳材料(SC) 是有效解决办法之一。近期的研究结果表明, 球形 碳材料(SC)具有锂离子扩散路径较短、比表面积大 等优势, 从而表现出高比容量和优异的倍率性能 ${ }^{[4]}$, 成为有可能取代石墨的商用负极材料。目前, 球形 碳材料的合成方法主要有电弧法、气相沉积法、水 热法及模板法等 ${ }^{[5-7]}$, 其中电弧放电法具有产物石 墨化程度高且可使用固体碳源的优势。此外, 为了满 足不断提高的锂离子电池负极材料的要求, 优化现 有球形碳材料的电化学性能, 必须对 SC 进行晶体结 构调变和表面改性等。 $\mathrm{SC}$ 的多孔化, 一般是通过使 用诸如 $\mathrm{KOH} 、 \mathrm{NaOH} 、 \mathrm{ZnCl}_{2} 、 \mathrm{H}_{3} \mathrm{PO}_{4} 、 \mathrm{CO}_{2}$ 和水蒸汽 等活化剂侵蚀碳的表面 ${ }^{[8-11]}$, 形成具有大孔、介孔、 微孔的分级多孔碳, 经造孔处理的碳材料比表面积 得到大幅度的提高, 可以提供更多的储锂活性位点。

本工作以新疆库车产煤为原料, 采用电弧放电 技术在 $\mathrm{He}$ 气氛下制备出粒径在 70 100 $\mathrm{nm}$ 的煤基 球形碳, 以不同浓度的 $\mathrm{KOH}$ 作为活化剂来构造煤基 球形碳(CSC)的多孔结构。在 $100 \mathrm{~mA} / \mathrm{g}$ 的电流密度 下对不同条件下获得的多孔化的 CSC 进行锂离子 电池充放电性能的测试, 以期获得性能最佳的锂电 负极用煤基球形碳材料。

\section{1 实验方法}

\section{1 主要原料和试剂}

煤, 产地新疆库车(煤样的工业分析及元素分 析见文献 $\left.{ }^{[12]}\right)$; 浓盐酸, 氢氟酸, 氢氧化钾, 乙炔黑, 聚偏氟乙烯(PVDF), $\mathrm{N}$-甲基吡咯烷酮 $\left(\mathrm{C}_{5} \mathrm{H}_{9} \mathrm{NO}\right)$, 均 为分析纯。高纯锂片, 电解液 LB-302, 高纯氦气。

\section{2 煤基球形碳的制备}

将经过盐酸及氢氟酸预处理的煤粉(直径 $<150 \mu \mathrm{m})$ 与煤焦油按一定比例(0.65:0.35)混和均匀 后, 使用电动等静压机在 $15 \sim 20 \mathrm{MPa}$ 的压力下压制 成直径约为 $10 \mathrm{~mm}$ 的煤棒。将压制成型的煤棒置
于管式炉内, 在 $\mathrm{N}_{2}$ 气氛下以 $5^{\circ} \mathrm{C} / \mathrm{min}$ 从室温升至 $500^{\circ} \mathrm{C}$ 并恒温 $180 \mathrm{~min}$, 然后以 $3^{\circ} \mathrm{C} / \mathrm{min}$ 升温至 $950^{\circ} \mathrm{C}$ 恒温 $180 \mathrm{~min}$, 自然冷却后得到炭化的煤基炭棒。通 过等离子体电弧放电技术, 以炭化后的煤基炭棒为 阳极, 以直径为 $8 \mathrm{~mm}$ 的石墨棒为阴极, 在氦气压 力为 $50 \mathrm{kPa}$ 、电压为 $30 \sim 40 \mathrm{~V}$ 、电流为 $90 \sim 100 \mathrm{~A}$ 的 条件下放电 $10 \mathrm{~min}$, 收集反应器顶部黑色粉末即为 煤基球形碳, 标记为 CSC。

分别称取 $100 \mathrm{mg}$ 的煤基球形碳置于浓度为 4、 $5 、 6$ 和 $7 \mathrm{~mol} / \mathrm{L}$ 的 $\mathrm{KOH}$ 溶液中, 室温下搅拌 $24 \mathrm{~h}$, 抽 滤、烘干后将其置于管式炉中, 在 $\mathrm{N}_{2}$ 气氛下 $900^{\circ} \mathrm{C}$ 处理 $2 \mathrm{~h}$ 。用 $3 \mathrm{~mol} / \mathrm{L}$ 的 $\mathrm{HCl}$ 和蒸馏水将活化产物洗 涤至中性, 再将其放置在烘箱中 $80^{\circ} \mathrm{C}$ 干燥 $12 \mathrm{~h}$, 所 得多孔煤基球形碳分别标记为 CSPC-1、CSPC-2、 CSPC-3、CSPC-4。

\section{3 产物的表征}

利用场发射扫描电镜(FESEM, Hitachi S-4800) 和透射电镜(TEM, Hitachi H-600)对产物的形貌进行 表征。在 X 射线衍射仪(XRD, Bruker D8)上测定样品 的物相结构, 以 $\mathrm{Cu} \mathrm{K \alpha}$ 为辐射源, 波长为 $0.15406 \mathrm{~nm}$, $2 \theta$ 角度扫描范围 $10^{\circ} \sim 80^{\circ}$; 采用激光显微共聚焦拉 曼光谱仪 (Raman, SENTERRA)检测碳球的结构信 息以及产物石墨化程度，激发光波长为 $532 \mathrm{~nm}$ 。采 用美国 Micromeritics ASAP $2020 \mathrm{~N}_{2}$ 吸脱附仪测试 样品的比表面积和孔径分布。在 Land 2001A 蓝电测 试仪上测试样品的充放电性能。在 CHI600D 电化学 工作站进行循环伏安测试, 电压范围 $0 \sim 3 \mathrm{~V}$, 扫描速 度 $0.1 \mathrm{mV} / \mathrm{s}$ 。在 Zahner Elektrik 电化学工作站进行 交流阻抗测试, 振幅 $5 \mathrm{mV}$, 频率 $0.01 \sim 100 \mathrm{kHz}$ 。

\section{2 结果与讨论}

\subsection{CSC 及 CSPC 的形貌与结构}

图 1(a)为 CSC 及活化后 CSPC-n 的 XRD 图谱, 由图可知, CSC 在 $2 \theta=22.8^{\circ}$ 和 $42.5^{\circ}$ 出现了两个宽的 衍射峰, 对应于具有六方点阵石墨的(002)和(100) 晶面 ${ }^{[5]}$, 但其石墨化程度较低, 这可能是样品中含 有大量无定形碳造成的。不同条件下获得的 CSPC- $n$ 均出现了对应于石墨炭(002)和(101)晶面的特征衍 射峰, 但在 $2 \theta=42.4^{\circ}$ 处对应于(100)晶面的衍射峰强 度变弱, 说明 CSC 经活化后, 微晶的层面堆叠有序 度降低。随着 $\mathrm{KOH}$ 溶液浓度的增大, CSPC $-n$ 的特 征衍射峰的位置未发生变化, 说明使用不同浓度的 活化剂活化不改变 CSPC 的结构。从图 1 还可以看 出, (002)晶面衍射峰的相对强度随 $\mathrm{KOH}$ 浓度的增 

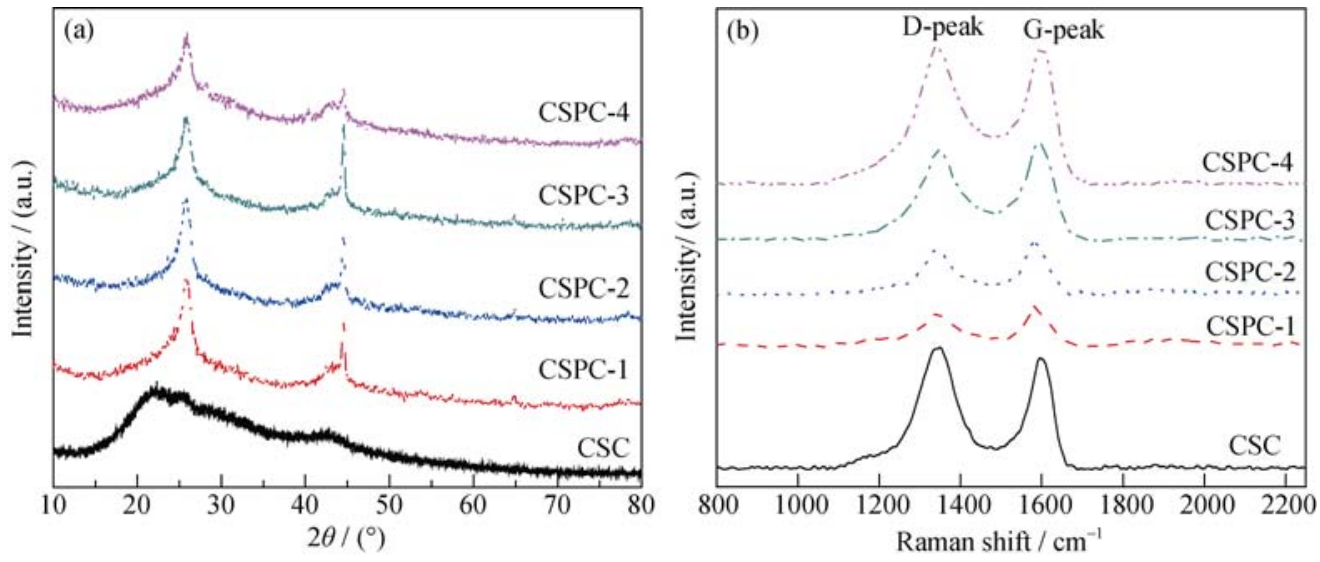

图 1 CSC 和 CSPC- $n$ 的(a)XRD 图谱, (b)Raman 图谱

Fig. 1 XRD patterns (a) and Raman spectra (b) of CSC and CSPC- $n$

大而减弱，表明 $\mathrm{KOH}$ 对煤基球形碳材料的刻蚀程 度增加, 致使 CSPC- $n$ 的石墨化程度逐渐降低。经布 拉格方程计算 CSPC- $n$ 的(002)晶面层间距分别为 $0.3497 、 0.3453 、 0.3447 、 0.3448 \mathrm{~nm}$ ，均大于结构完 美的石墨晶体的层间距 $(0.3354 \mathrm{~nm})$, 说明 CSPC- $n$ 的石墨化程度较低。

不同浓度 $\mathrm{KOH}$ 活化所得 CSPC- $n$ 的 Raman 光谱 如图 1(b)所示, 图中波数为 $1345 \mathrm{~cm}^{-1}$ 的吸收峰(称为 D 峰)主要是由碳材料缺陷引起的, 对应于无序石墨 层内的 $\mathrm{C}-\mathrm{C}$ “悬荡”键的振动吸收; 波数为 $1580 \mathrm{~cm}^{-1}$ 的吸收峰(称为 $\mathrm{G}$ 峰), 对应于石墨 $\mathrm{E}_{2 \mathrm{~g}}$ 振动模式, 它与 二维石墨层内 $\mathrm{sp}^{2}$ 杂化碳原子振动吸收有关。 $\mathrm{G}$ 峰和 $\mathrm{D}$ 峰的相对强度比值 $\left(I_{\mathrm{G}} / I_{\mathrm{D}}\right)$ 常用来描述碳材料结构中 缺陷的密集度, $I_{\mathrm{G}} / I_{\mathrm{D}}$ 值越大, 表明碳材料表面的缺陷 越少 ${ }^{[13]}$ 。由图 1 可知, 随着 $\mathrm{KOH}$ 溶液浓度的增大, $\mathrm{CSPC}$ 的 $I_{\mathrm{G}} / I_{\mathrm{D}}$ 值依次为: $1.07(\mathrm{CSPC}-1), 1.05(\mathrm{CSPC}-2)$,
1.02(CSPC-3)和 0.99(CSPC-4), 表明随着 $\mathrm{KOH}$ 浓度的 增大, CSPC 的有序度逐渐降低, 表面的缺陷逐渐增多, 石墨化程度不断降低, 这与 XRD 的分析结果一致。

由 CSC 的 FESEM 照片(图 2(a))可以看出, CSC 为球形颗粒，粒径分布不均匀，颗粒之间有明显团 聚现象并存在一些无规则形貌的碳颗粒; 图 2(b) (e) 为不同浓度 $\mathrm{KOH}$ 活化后所得 CSPC- $n$ 的 FESEM 照 片, 随着 $\mathrm{KOH}$ 溶液浓度增大, 无规则形貌碳颗粒减 少，球形颗粒尺寸分布均匀度增加，颗粒之间的团 聚有所改善, 但仍然存在粘连现象; 当 $\mathrm{KOH}$ 浓度增 大到 $7 \mathrm{~mol} / \mathrm{L}$ 时(图 2(e)), 球形颗粒之间再次发生明 显团聚, 这可能是由于 $\mathrm{KOH}$ 浓度过大时, 球形结构 发生过度刻蚀造成部分碳球结构妇塌。样品 CSPC-3 的 TEM 形貌如图 2(f)所示, 从图中可以看出产物 呈球形，粒径为 70 100 nm, 球形颗粒之间有团聚, 这与 FESEM 分析结果一致。
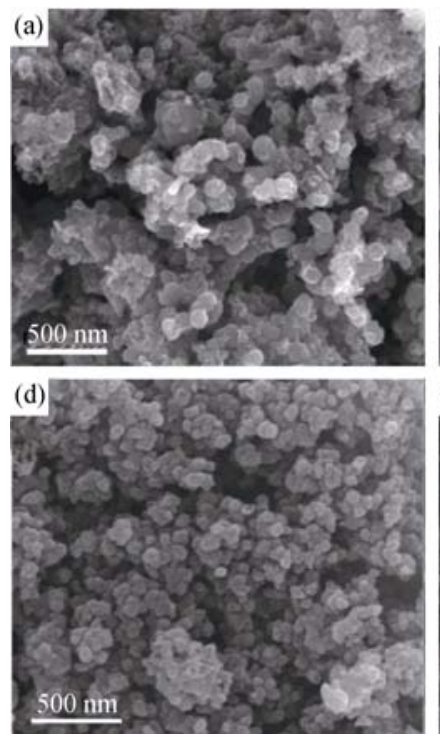
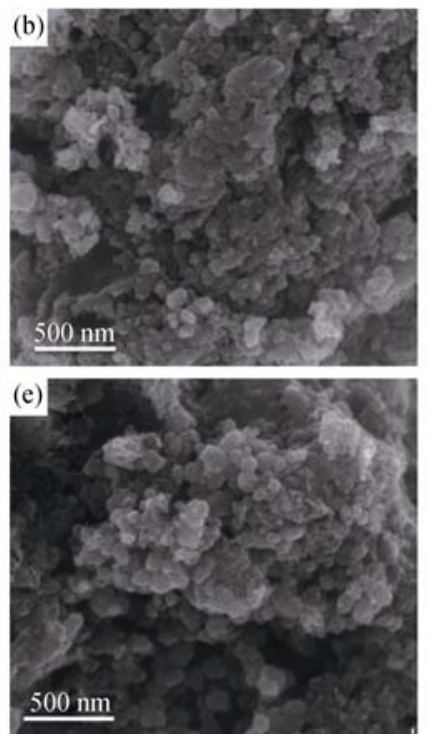
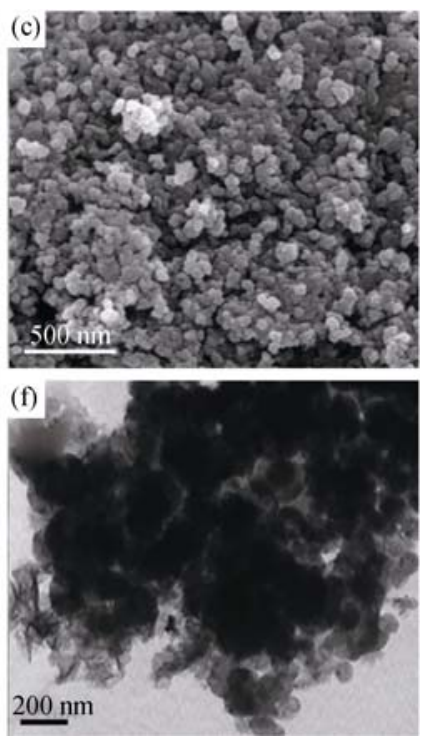

图 2 (a)CSC,(b)CSPC-1,(c)CSPC-2, (d)CSPC -3 和(e)CSPC-4 的 FESEM 照片以及(f)CSPC-3 的 TEM 照片

Fig. 2 FESEM images of (a) CSC, (b) CSPC-1, (c) CSPC-2, (d) CSPC-3, and (e) CSPC-4, and the TEM image of CSPC-3 (f) 
图 3(a e) 为 CSC 及 CSPC- $n$ 的氮气吸脱附等温 线。CSC 的吸附曲线呈现出典型的 III 型吸附曲线 特征, 随着相对压力的升高, 吸附量增大。与未经 活化的 CSC 相比, 经不同浓度 $\mathrm{KOH}$ 活化的 CSPC- $n$ 在较低的相对压力下有明显的吸附增量, 说明活化处理产生了大量的微孔结构, 其吸附曲 线在相对压力为 0.4 时出现毛细管凝聚现象, 呈现 出典型的 IV 型吸附曲线及 $\mathrm{H} 4$ 型滞后回线, 说明活 化后 CSC 具有微孔和介孔结构 ${ }^{[14]}$ 。由氮气吸脱附 等温线得到材料的孔结构参数及比表面积数据列 于表 1 中。随着 $\mathrm{KOH}$ 溶液浓度的增大, CSPC- $n$ 的 比表面积逐渐增大, 可由 $117.17 \mathrm{~m}^{2} / \mathrm{g}(\mathrm{CSC})$ 增加至
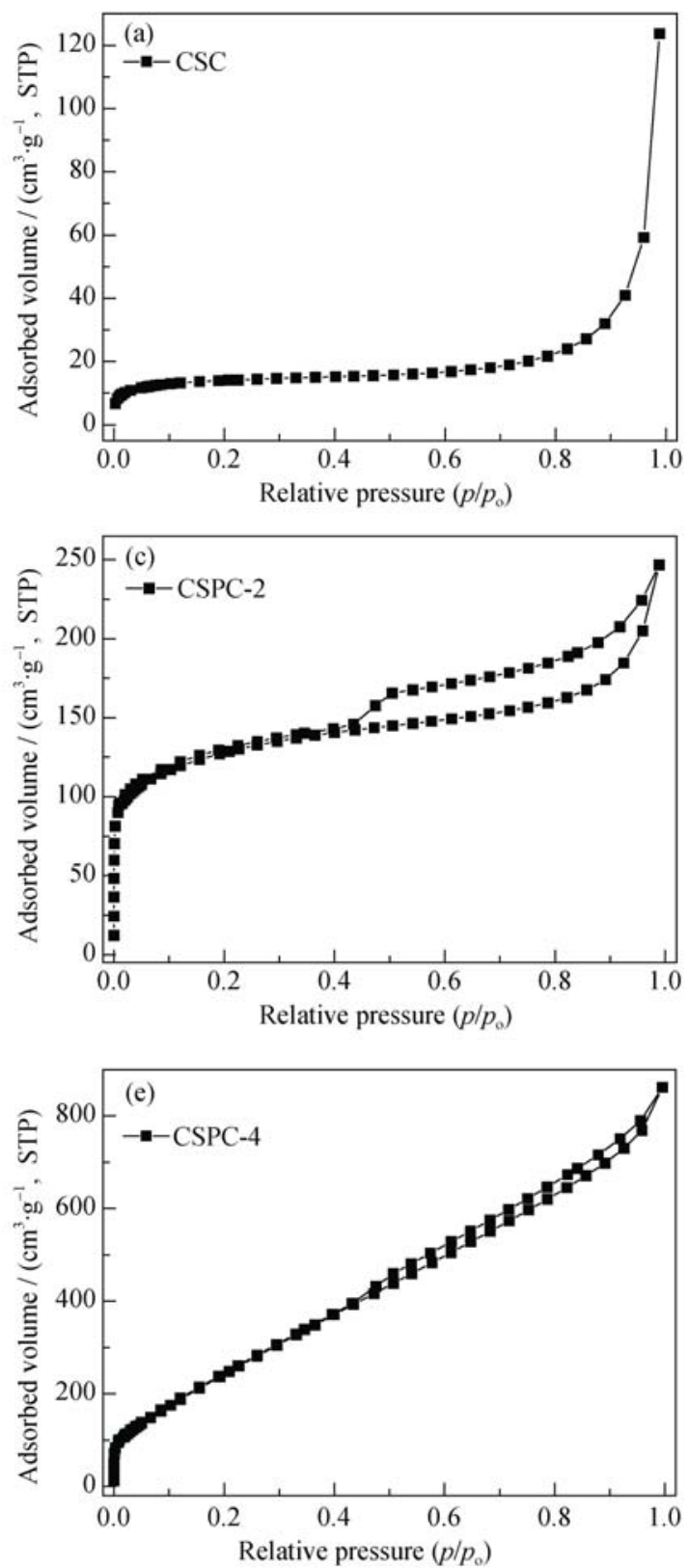

$810.42 \mathrm{~m}^{2} / \mathrm{g}$ (CSPC-4)。DFT 孔径分布曲线表明(图 $3 \mathrm{f}), \mathrm{CSC}$ 的孔径分布主要集中在 2.7 62.6 nm, 主 要以介孔和大孔为主; 当 $\mathrm{KOH}$ 的浓度小于 $7 \mathrm{~mol} / \mathrm{L}$ 时, CSPC- $n(n=1,2,3)$ 孔径分布呈现出既有微孔、介 孔又有大孔的分级孔道结构, 并且随着 $\mathrm{KOH}$ 溶液 浓度的增大，其孔径呈增大趋势; 当 $\mathrm{KOH}$ 溶液浓度 增大到 $7 \mathrm{~mol} / \mathrm{L}$ 时, CSPC-4 中的微孔基本消失，而 介孔则大幅增加，孔径分布集中在 $2.7 \sim 87 \mathrm{~nm}$ ，这 说明 $\mathrm{KOH}$ 浓度过高时, 活化所产生的微孔在大量 强碱的作用下被刻蚀成介孔或大孔，表 1 中 CSPC-4 的微孔孔容数据也进一步说明其微孔的 减少。
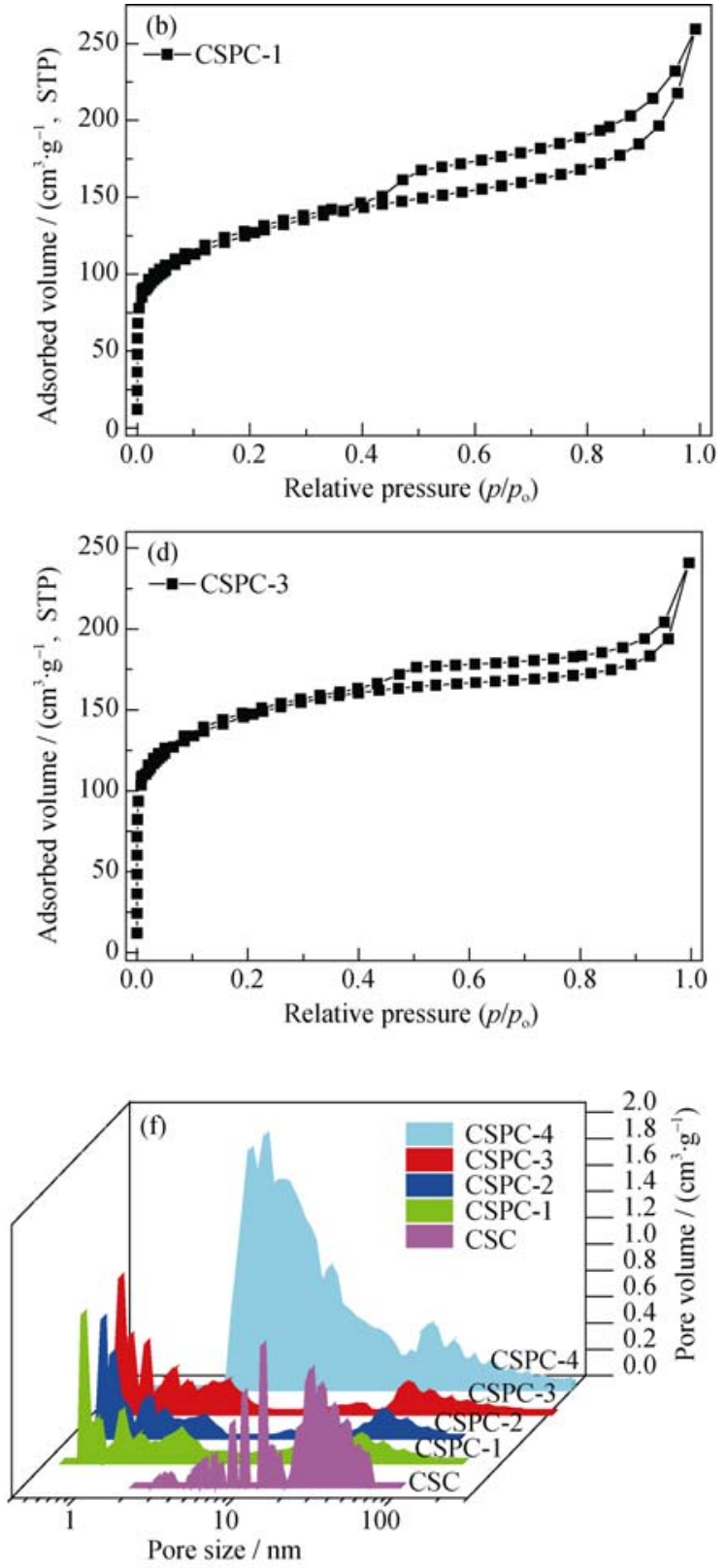

图 3 (a e)CSC 及 CSPC-n 的氮气吸脱附等温线, (f)CSC 及 CSPC- $n$ 的 DFT 孔径分布图

Fig. 3 Nitrogen sorption isotherms of (a-e) CSC and CSPC-n, (f) DFT pore size distributions of CSC and CSPC- $n$ 
表 1 CSC 及 CSPC-n 的比表面积和孔结构参数

Table 1 The pore structure parameters of CSC and CSPC-n

\begin{tabular}{ccccc}
\hline Sample & $\begin{array}{c}S_{\mathrm{BET}} \\
/\left(\mathrm{m}^{2} \cdot \mathrm{g}^{-1}\right)\end{array}$ & $\begin{array}{c}V_{\mathrm{BJH}} \\
/\left(\mathrm{cm}^{3} \cdot \mathrm{g}^{-1}\right)\end{array}$ & $\begin{array}{c}V_{\text {micro }} \\
/\left(\mathrm{cm}^{3} \cdot \mathrm{g}^{-1}\right)\end{array}$ & $\left(V_{\text {micro }} / V_{t}\right) / \%$ \\
\hline CSC & 117.17 & 0.35 & 0.01 & 3.48 \\
CSPC-1 & 442.81 & 0.40 & 0.07 & 17.44 \\
CSPC-2 & 464.18 & 0.44 & 0.08 & 18.05 \\
CSPC-3 & 534.83 & 0.37 & 0.12 & 31.83 \\
CSPC-4 & 810.42 & 1.33 & 0.31 & 23.30 \\
\hline
\end{tabular}

\subsection{CSC 与 CSPC 的电化学性能}

图 4 是在 $100 \mathrm{~mA} / \mathrm{g}$ 的电流密度下 $\mathrm{CSC}$ 和 CSPC- $n$ 的首次和第二次充放电曲线, 从图中可以 看出, CSC 和 CSPC- $n$ 在低电压区间都存在一个明显 的斜坡，这与文献报道的碳纳米管、碳纤维及无定形 碳材料的放电曲线类似 ${ }^{[15-17]}$ 。CSC 与 CSPC- $n$ 的充放 电曲线相似，五个样品有着相同的储锂机制，其首 次充放电效率均较低, 这与放电曲线上 $0.9 \sim 0.7 \mathrm{~V}$ 间 平台所对应的 SEI(solid electrolyte interface)膜

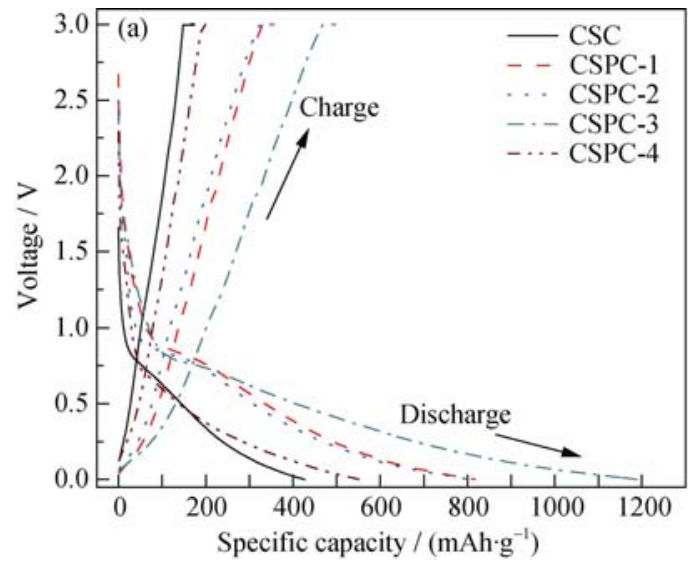

的形成有关 ${ }^{[18]}$ 。在第二次放电曲线中平台消失，放 电曲线呈现“大斜坡”线型，说明 SEI 膜的形成只存 在于首次放电过程中。 $\mathrm{CSC}$ 和 CSPC- $n$ 的首次和第 二次充、放电比电容量及首次库伦效率数据列于表 2 中。由表 2 数据可知, CSPC-3 的首次充放电容量 明显高于其他材料。

CSPC- $n$ 的循环性能如图 5 所示, 由图可知, CSPC- $n$ 在前 20 圈容量都有不同程度的衰减，这可 能是循环中电极材料体积的变化所导致的, 但在随 后的放电过程中逐渐稳定并得到修复。尤其是 CSPC-3 由首圈的 $1188.9 \mathrm{mAh} / \mathrm{g}$ 衰减至第 20 圈的 $398.4 \mathrm{mAh} / \mathrm{g}$, 但在随后的长程充放电过程中容量得 到了提高, 200 圈充电后甚至达到 $844.9 \mathrm{mAh} / \mathrm{g}$, 其 库伦效率也在充放电 20 圈后保持在 $98.5 \%$ 以上。放 电比容量随着充放电次数的增加呈现上升趋势的现 象在其他碳纳米材料中也有出现 ${ }^{[19-21]}$, 这可能是由 于电极材料具有多孔结构, 随着循环次数的增加, 电解液与碳材料得到充分的接触, 促进了表面电荷 的传输, 缩短了锂离子的传输路径, 并为锂离子的 存储提供更多的空间，从而增大了容量 ${ }^{[22]}$ 。

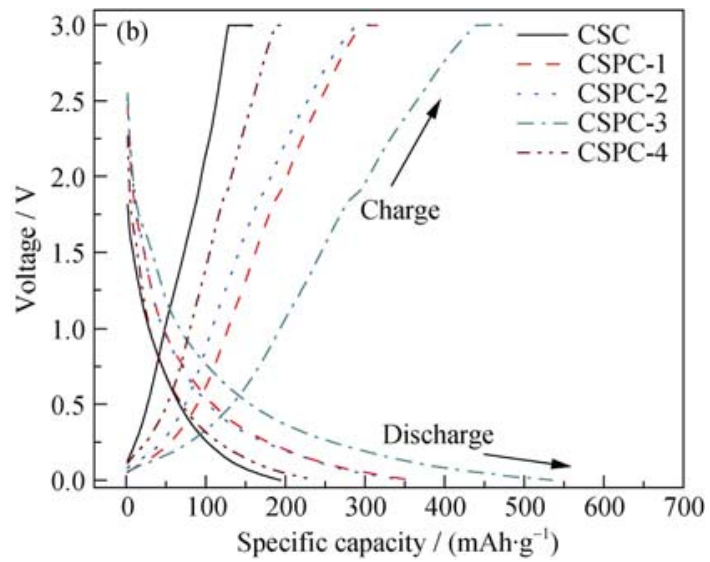

图 4 CSC 及 CSPC- $n$ 的首次(a)和第二次(b)充放电曲线

Fig. 4 Charge/discharge curves in the first cycle (a) and (b) the second cycle of CSC and CSPC-n electrodes

表 2 CSPC-n 电极的充放电数据

Table 2 Charge/discharge capacity and the efficiency of the CSPC-n electrodes

\begin{tabular}{ccccccccc}
\hline Sample & $\begin{array}{c}\text { First } \\
\text { specific } \\
\text { discharge } \\
\text { capacity } \\
/\left(\mathrm{mAh}^{-1}\right)\end{array}$ & $\begin{array}{c}\text { First } \\
\text { specific } \\
\text { charge } \\
\text { capacity } \\
/\left(\mathrm{mAh} \cdot \mathrm{g}^{-1}\right)\end{array}$ & $\begin{array}{c}\text { First } \\
\text { coulombic } \\
\text { efficiency } / \%\end{array}$ & $\begin{array}{c}\text { Second } \\
\text { specific } \\
\text { discharge } \\
\text { capacity } \\
/\left(\mathrm{mAh} \cdot \mathrm{g}^{-1}\right)\end{array}$ & $\begin{array}{c}\text { Second } \\
\text { specific } \\
\text { charge } \\
\text { capacity } \\
/\left(\mathrm{mAh} \cdot \mathrm{g}^{-1}\right)\end{array}$ & $\begin{array}{c}\text { Second } \\
\text { coulombic } \\
\text { efficiency/\% }\end{array}$ & $\begin{array}{c}\text { The 20th } \\
\text { specific } \\
\text { discharge } \\
\text { capacity }\end{array}$ & $\begin{array}{c}\text { The 20th } \\
\text { specific } \\
\text { charge } \\
\text { capacity } \\
/\left(\mathrm{mAh} \cdot \mathrm{g}^{-1}\right)\end{array}$ \\
$/\left(\mathrm{mAh} \cdot \mathrm{g}^{-1}\right)$
\end{tabular}




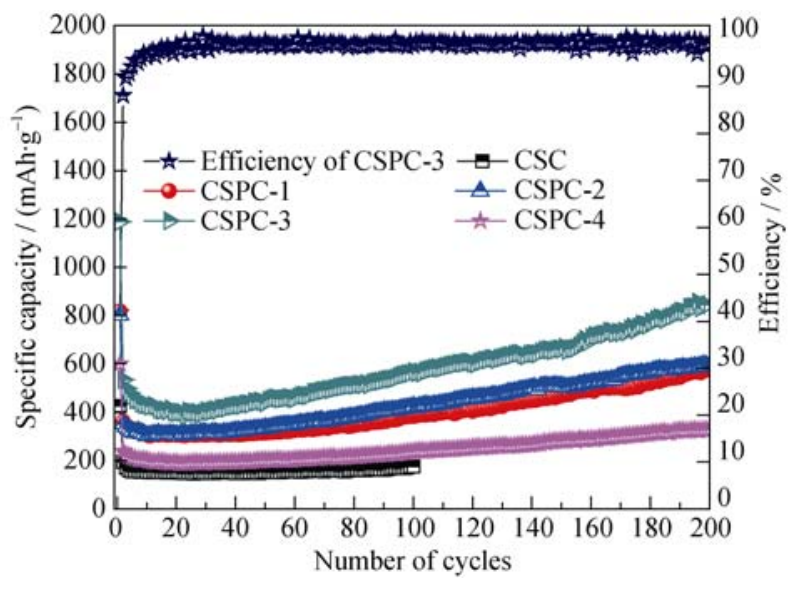

图 $5 \mathrm{CSC}$ 及 CSPC- $n$ 的容量循环次数关系图

Fig. 5 Cyclic performance of CSC and CSPC- $n$ electrodes

基于 CSPC-3 较优异的电化学性能, 我们对其 电化学行为进行了更深入的研究。CSPC-3 的 CV 测 试如图 6(a)所示, 首圈循环曲线的氧化峰面积比还 原峰面积明显减小, 说明其氧化还原过程有一定不 可逆性, 造成首次库伦效率较低。在首圈放电过程 中, 在 $0.7 \mathrm{~V}$ 附近出现了一个还原峰, 对应于 SEI 膜 的形成过程，与首次充放电曲线中的电位拐点相对 应; 而在 $0.01 \mathrm{~V}$ 附近的还原峰较强, 对应于锂离子嵌 入到碳层中 ${ }^{[23]}$ 。在接下来的循环扫描中, 位于 $0.7 \mathrm{~V}$ 附近的还原峰消失, 说明首次循环过程中 SEI 膜已 稳定形成, 不再需要耗费锂离子和电解液, 这与恒 流充放电的测试结果相符合。从图 6(a)插图中可以 看出, 在 $0.17 \mathrm{~V}$ 附近对应于锂离子在碳层内的脱出 反应的氧化峰, 随着循环次数的增加, 峰强和峰面 积有所增加, 不可逆容量减少, 循环稳定性增加。 CSPC-3 的交流阻抗测试(EIS)如图 6(b)所示, 其电 化学交流阻抗曲线由高频区的半圆弧和低频区的斜 线组成。一般认为锂离子电池负极 Nyquist 曲线的

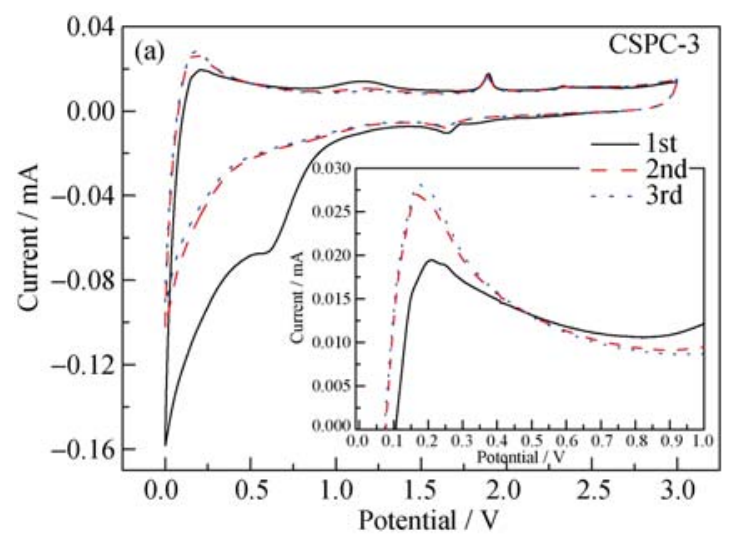

半圆弧的直径与 SEI 膜及电荷转移电阻的大小等因 素有关 ${ }^{[24]}$, 斜线则代表着锂离子在负极材料中有限 扩散的类 Warburg 阻抗 ${ }^{[25]}$ 。CSPC-3 电极在高频区 的圆弧半径值小于 $\mathrm{CSC}$ 电极, 表明它具有更好的电 导率和更小的材料间或材料和集流体间的接触电阻, 可以有效加快锂离子在嵌入与脱出过程中电荷的迁 移速率; 低频区的阻抗曲线斜率较大, 表明 CSPC-3 电极与电解液之间有较高的传质速率 ${ }^{[26]}$ 。

CSPC-3 电极的电化学性能优异可能是由于: (1) 经活化后的煤基球形碳的比表面积较大，提供了更 多可用于储锂的活性位点，同时也增加了碳材料与 电解液的接触面积，增大了碳材料的利用率; (2)基于 微孔储锂理论 ${ }^{[27]}$, 活化后的样品产生了大量的微孔 和介孔，为锂离子的储存提供了更多的空间，因而 获得了较高的比容量。

\section{3 结论}

以新疆库车产煤为原料, 采用电弧放电技术制备 了煤基球形碳材料, 并通过不同浓度的 $\mathrm{KOH}$ 溶液对 球形碳材料进行活化。测试结果表明, 随着 $\mathrm{KOH}$ 溶 液浓度的增大, 样品的石墨化程度逐渐降低, 比表面 积由 $117.17 \mathrm{~m}^{2} / \mathrm{g}$ (CSC)增加到 $810.42 \mathrm{~m}^{2} / \mathrm{g}$ (CSPC-4)。 此外, 通过改变 $\mathrm{KOH}$ 浓度还可实现样品表面孔隙结 构的调控: 使用中低浓度 $(4 \sim 6 \mathrm{~mol} / \mathrm{L})$ 的 $\mathrm{KOH}$ 溶液可 得到具有微孔、介孔又有大孔的分级孔道结构碳球; 在高浓度 $\mathrm{KOH}$ 溶液中 $(7 \mathrm{~mol} / \mathrm{L})$, 样品表面的微孔基 本消失, 而介孔则大幅增加。电化学性能测试表明, 样品的电化学性能并非随着比表面积的增加而增大, 而与样品的孔隙结构密切相关。当样品具有丰富微 孔和介孔结构时，可为锂离子提供更多储存空间， 从而提高了样品的容量及循环稳定性。通过制备

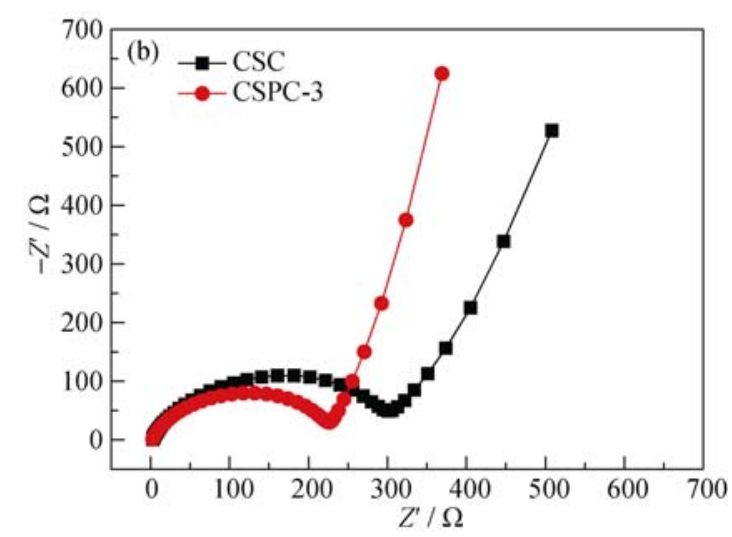

图 6 (a) CSPC-3 的循环伏安曲线(内插图为局部放大图)和(b)CSPC-3 的交流阻抗曲线

Fig. 6 (a) Cyclic voltammetry curves of CSPC-3 electrodes (insert showing the detail with enlarged scale) and (b) Nyquist plots for CSPC- $n$ electrodes 
条件的优化, 样品(CSPC-3)在 $100 \mathrm{~mA} / \mathrm{g}$ 的电流密度

下，首次放电比容量可达到 $1188.9 \mathrm{mAh} / \mathrm{g}$, 经过 200

圈充放电循环后库伦效率保持在 $98.5 \%$ 以上。以煤 为原料制备具有优异电化学性能的功能碳材料, 不 仅可以为储量丰富的煤炭资源转化为功能碳材料奠 定技术基础, 而且为煤炭资源的高附加值加工利用 提供了新途径。

\section{参考文献:}

[1] TARASCON J M, ARMAND M. Issues and challenges facing rechargeable lithium batteries. Nature, 2001, 414: 359-367.

[2] WU Y P, MA J Q, DAI X B, et al. Lithium Ion Batteries: Practice Applications. Chemical Industry Press, Beijing, 2004.

[3] SU L, JING Y, ZHOU Z. Li ion battery materials with core-shell nanostructures. Nanoscale, 2011, 3(10): 3967-3983.

[4] ROBERTS A D, LI X, ZHANG H F. Porous carbon spheres and monoliths: morphology control, pore size tuning and their applications as Li-ion battery anode materials. Chem. Soc. Rev., 2014, 43(34): 4341-4356.

[5] JIN Y Z, GAO C, HSU W K, et al. Large-scale synthesis and characterization of carbon spheres prepared by direct pyrolysis of hydrocarbons. Carbon, 2005, 43(9): 1944-1953.

[6] LI M, LI W, LIU S X. Hydrothermal synthesis, characterization, and $\mathrm{KOH}$ activation of carbon spheres from glucose. Carbohydr. Res., 2011, 346(8): 999-1004.

[7] TIEN B, XU M, LIU J. Synthesis and electrochemical characterization of carbon spheres as anode material for lithium-ion battery. Mater. Lett., 2010, 64(13): 1465-1467.

[8] CHOI M, RYOO R. Mesoporous carbons with $\mathrm{KOH}$ activated framework and their hydrogen adsorption. J. Mater. Chem., 2007, 17(39): 4204-4309.

[9] ZHU Y, MURALI S, STOLLER M D, et al. Carbon-based supercapacitors produced by activation of graphene. Science, 2011, 332(6037): 1537-1541.

[10] CHANG B, SHI W, GUAN D, et al. Hollow porous carbon sphere prepared by a facile activation method and its rapid phenol removal. Mater. Lett., 2014, 126(126): 13-16.

[11] LI W J, YU Z Y, XIE F, et al. Preparation and characterization of spherical mesoporous material SBA-15 in the presence of $\mathrm{H}_{3} \mathrm{PO}_{4}$. Mater. Sci. Forum, 2011, 694: 804-808.

[12] WU X, WANG L X, JIA D Z. Controllable preparation of carbon nanotubes from xinjiang coal. Chinese J. Inorg. Chem., 2013, 29(9): 1842-1848.
[13] DRESSELHAUS M S, DRESSELHAUS G, SAITO R, et al. Raman spectroscopy of carbon nanotubes. Phys. Rep., 2005, 409(2): 47-99.

[14] KONNDOU S, ISHIKAWA T, ABE I. Franslated by LI G X. Adsorption Science. Chemical Industry Press, Beijing, 2006.

[15] WANG S X, YANG L, STUBBS L P, et al. Lignin-derived fused electrospun carbon fibrous mats as high performance anode materials for lithium ion batteries. ACS Appl. Mater. Interfaces, 2013, 5(23): 12275-12282.

[16] QIAN C, GUO P, ZHANG X, et al. Nitrogen-doped mesoporous hollow carbon nanoflowers as high performance anode materials of lithium ion batteries. RSC Adv., 2016, 6: 93519-93524.

[17] ZHONG C, WANG J Z, WESLER D, et al. Microwave autoclave synthesized multi-layer graphene/single-walled carbon nanotube composites for free-standing lithium-ion battery anodes. Carbon, 2014, 66: 637-645.

[18] CHEN Y M, LI X Y, PARK K. Hollow carbon-nanotube/carbonnanofiber hybrid anodes for Li-ion batteries. J. Am. Chem. Soc., 2013, 135(44): 16280-16283.

[19] WU P, DU N, ZHANG H, et al. Carbon nanocapsules as nanoreactors for controllable synthesis of encapsulated iron and iron oxides: magnetic properties and reversible lithium storage. $J$. Phy. Chem. C, 2011, 115(9): 3612-3620.

[20] LI X N, ZHU X B, ZHU Y C, et al. Porous nitrogen-doped carbon vegetable-sponges with enhanced lithium storage performance. Carbon, 2014, 69(2): 515-524.

[21] TANG J J, YANG J, ZHOU X Y, Synthesis and characterisation of sponge-like carbon anode materials for lithium ion batteries. Mater. Lett., 2013, 109(15): 253-256.

[22] SAIKIA D, WANG T H, CHOU C J, et al. A comparative study of ordered mesoporous carbons with different pore structures as anode materials for lithium-ion batteries. RSC $A d v$., 2015, 5(53): 42922-42930.

[23] NITIN A K, JOACHIM M. Lithium storage in carbon nanostructures. Adv. Mater, 2009, 21(25/26): 2664-2680.

[24] GAN L, GUO H J, WANG Z X, et al. A facile synthesis of graphite/silicon/graphene spherical composite anode for lithium-ion batteries. Electrochim. Acta., 2013, 104(8): 117-123.

[25] CHANG Y C, SOHN H J. Electrochemical impedance analysis for lithium ion intercalation into graphitized carbons. J. Electrochem. Soc., 2000, 147: 50-58.

[26] XING Z, JU Z C, ZHAO Y L, et al. One-pot hydrothermal synthesis of nitrogen-doped graphene as high-performance anode materials for lithium ion batteries. Sci. Rep., 2016, 6: 26146.

[27] WU Y P, WAN C R, JIANG C Y, et al. Mechanism of lithium storage in low temperature carbon. Carbon, 1999, 37: 1901-1908. 